\title{
In Situ Transmission Electron Microscopy Imaging of Electromigration in Platinum Nanowires
}

\author{
Maria Rudneva, ${ }^{\star}$ Bo Gao, ${ }^{\dagger}$ Ferry Prins, Qiang Xu, Herre S.J. van der Zant, and \\ Henny W. Zandbergen \\ Kavli Institute of Nanoscience, Delft University of Technology, Lorentzweg 1, 2628 CJ Delft, The Netherlands
}

\begin{abstract}
In situ transmission electron microscopy was performed on the electromigration in platinum $(\mathrm{Pt})$ nanowires (14 nm thick, $200 \mathrm{~nm}$ wide, and $300 \mathrm{~nm}$ long) with and without feedback control. Using the feedback control mode, symmetric electrodes are obtained and the gap usually forms at the center of the Pt nanowire. Without feedback control, asymmetric electrodes are formed, and the gap can occur at any position along the wire. The three-dimensional gap geometries of the electrodes in the Pt nanowire were determined using high-angle annular dark-field scanning transmission electron microscopy; the thickness of the nanowire is reduced from $14 \mathrm{~nm}$ to only a few atoms at the edge with a gap of about 5-10 nm.
\end{abstract}

Key words: electromigration, transmission electron microscopy, in situ imaging

\section{INTRODUCTION}

Electromigration is a mass transport phenomenon induced by the impulse of charge carriers to lattice ions. Electromigration occurs when an electrical current with a density higher than the threshold is applied and is considered to be a failure mechanism in integrated circuits. Whereas electromigration can be detrimental for contact lines in ICs, it can be used to create nanogaps to determine the electronic properties of particles or molecules trapped in these nanogaps (Park et al., 1999). For example, a gap $\sim 1 \mathrm{~nm}$ wide has been fabricated using this technique to contact colloidal cadmium selenide nanocrystals.

When fabricating nanogaps to determine the electronic properties of trapped species, good control over the shape and size of the gap is essential. If one uses the conventional electromigration technique of uniform voltage increase, the formation of the nanogaps cannot be controlled: one typically gets a cascade of unpredictable changes in the nanowire. Feedback-controlled electromigration has been developed to get a better control of gap formation, whereby the current is reduced or switched off as soon as a sudden change in resistance is measured (Strachan et al., 2005; O'Neill et al., 2007). Finally, self-breaking schemes have been developed to avoid the formation of spurious metal grains (Houck et al., 2005; van der Zant et al., 2006) and high temperatures during the trapping of the molecules (O’Neill et al., 2007; Prins et al., 2009).

Scanning electron and transmission electron microscopy (TEM) imaging have been used to probe the gap formation with a high spatial resolution (Heersche et al., 2007; Strachan et al., 2008). A temperature rise during electromigration has also been reported (Esen \& Fuhrer, 2005; Taychatanapat et al., 2007). Previous publications on the imaging

\footnotetext{
() MICROSCOPY SOCIETY OF AMERICA 2013

${ }^{\star}$ Corresponding author. E-mail: M.Rudneva@tudelft.nl

${ }^{\dagger}$ Current address: Max Planck Institute for Solid State Research, Heisenbergstrasse 1, D-70569 Stuttgart, Germany
}

of nanogaps have focused on the electromigration of gold nanowires because gold is often used in molecular electronics (Park et al., 1999; Heersche et al., 2007). Recently, platinum $(\mathrm{Pt})$ has been considered as an alternative material, because it produces more stable nanoelectrodes than gold (Grose et al., 2008; Prins et al., 2009). Direct observation of electromigration in Pt nanowires has not been reported.

In this paper we present an in situ TEM study of electromigration in $\mathrm{Pt}$ nanowires. We compare the gap geometry realized with and without feedback control (FBC). Furthermore, the stability of the in situ measurement setup allows a more detailed investigation of the gap with various TEM techniques and the imaging of the self-breaking process of a Pt nanojunction with atomic resolution. We have also used scanning TEM (STEM) imaging to probe the height profile of the nanogap, thereby providing a threedimensional (3D) description of the geometry of the electrodes formed by electromigration.

\section{Materials and Methods}

Pt nanowires were patterned on 100-nm-thick silicon nitride membrane substrates using e-beam lithography (Gao et al., 2009). The Pt wire was $300 \mathrm{~nm}$ long and had a cross-section (width $\times$ thickness) of $200 \times 14 \mathrm{~nm}^{2}$. The total resistance of each device, including the resistance of the leads and the contact pads, was between 150 and $300 \Omega$. Before loading into the TEM, the membrane substrate was thinned from the back to a thickness of about $20 \mathrm{~nm}$ using reactive ion etching in order to reduce image deterioration by the amorphous silicon nitride membrane.

Imaging was performed with the Titan FEI microscope at $300 \mathrm{kV}$. The experiments were carried out at temperatures close to $100 \mathrm{~K}$ in order to reduce carbon contamination. A special sample holder built in-house has eight feedthroughs for performing electrical measurements. The construction of the holder allows the chip-like samples to be connected with the measurement setup. 


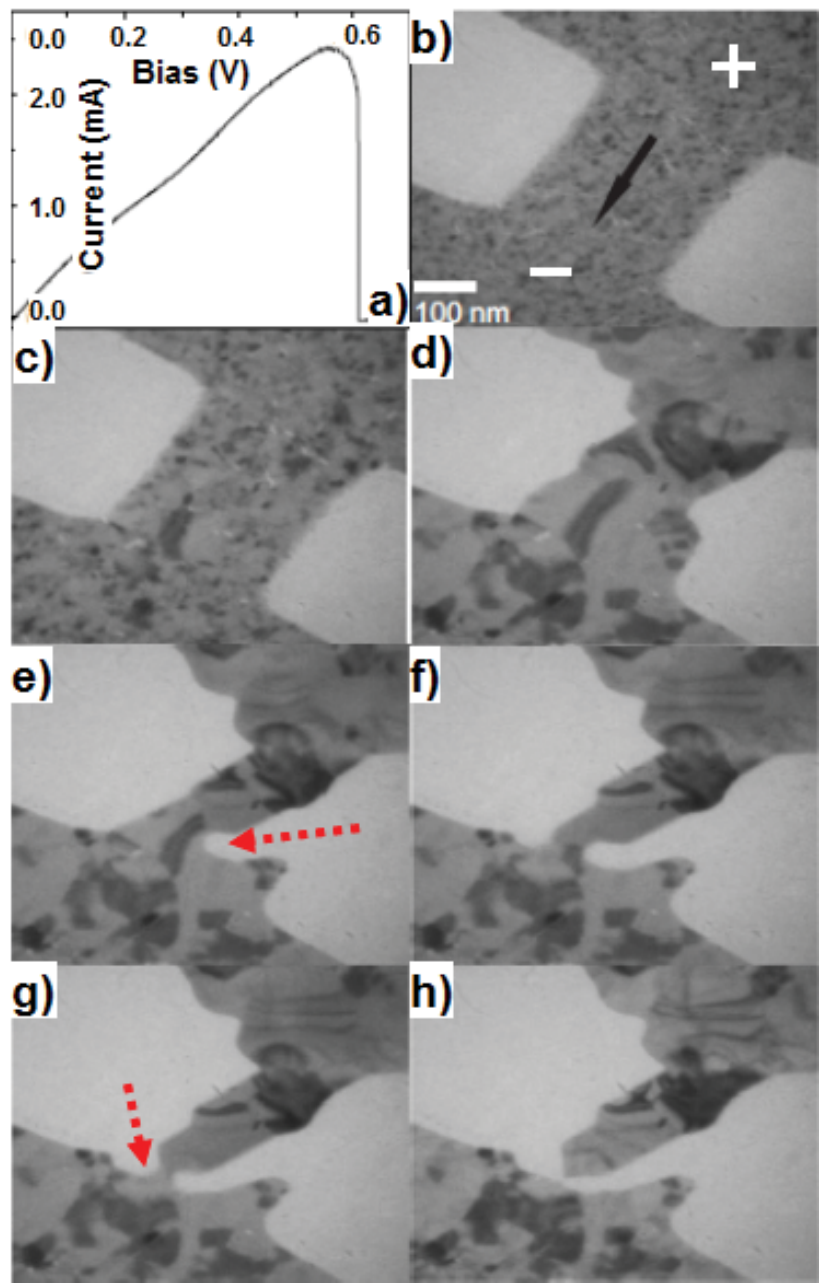

Figure 1. a: A current-voltage curve of an electromigration process without feedback control. The drop of the current signifies the breaking of the wire. $\mathbf{b}-\mathbf{h}$ : In situ transmission electron microscopy imaging of the electromigration. $\mathbf{b}$ : The platinum nanowire as fabricated; (c) grain growth taking place in the wire. The rest of the images show that the bottleneck of the wire shifts from the anode to cathode side, where the final gap is formed. The gap electrodes are asymmetrical. The arrow in (b) denotes the direction of the current. The scale bar applies to all the figures.

We performed in situ TEM electromigration using two different techniques: a mode with a uniform increase of the voltage (UVI) and a FBC mode. In the UVI mode, the bias voltage applied to the $\mathrm{Pt}$ nanowire increases slowly and linearly with time at a rate of about $8.3 \mathrm{mV} / \mathrm{s}$ until the wire is broken. A typical breakdown value for the presented samples is a few hundred millivolts. In FBC mode, the wire conductance is constantly monitored while the voltage is sweeping up. If there is a sudden decrease in conductance, the feedback control mechanism reduces the voltage to zero. The process starts again after we have set a new reference conductance. The program stops when a predefined conductance value is reached. We investigated a total of 12 devices with UVI and 16 devices with the FBC mode. All electrical measurements were combined with a simultaneous observation of the changes in the wire using a fast scan camera.
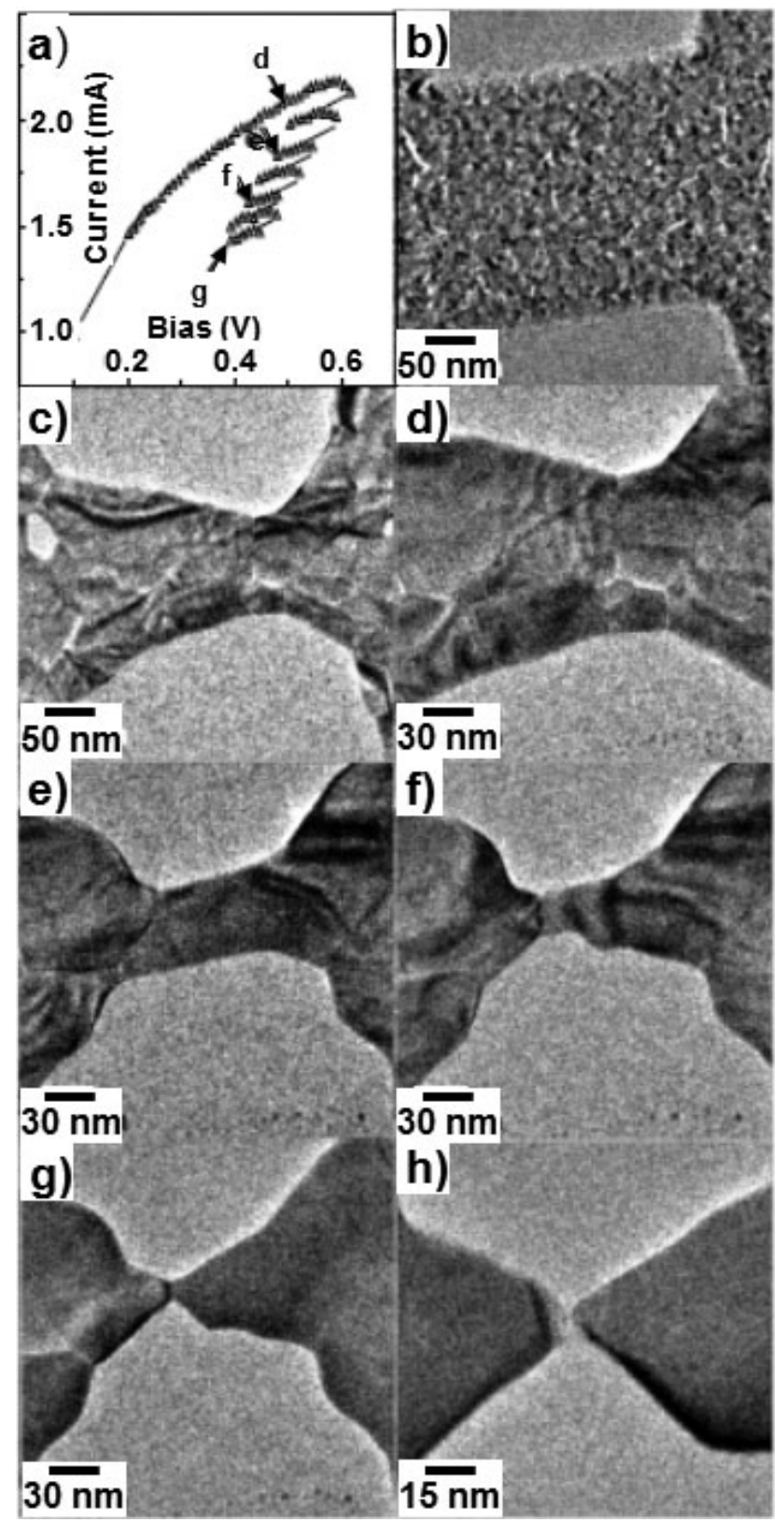

Figure 2. a: A current-voltage curve of an electromigration process with feedback control (FBC). Triangles indicate the measured I-V data points; the black lines signify the occurrence of the feedback loop. The I-V curve corresponds to the electromigration from image $(\mathbf{d}-\mathbf{g})$. More precisely, the four locations indicated by the arrow and letter correspond to the respective images below. b-h: In situ transmission electron microscopy imaging of the electromigration. $\mathbf{b}$ : The original platinum nanowire. The rest of the images show that FBC leads to symmetric electrodes on both sides of the final gap, and the gap in FBC usually appears near the center of the wire.

\section{RESULTS}

Figures 1 and 2 show typical video footage of the gap formation in UVI and FBC modes. In both cases, the initial Pt wire consists of small grains with an average diameter of $4 \mathrm{~nm}$. In addition, in both cases grain growth is observed 


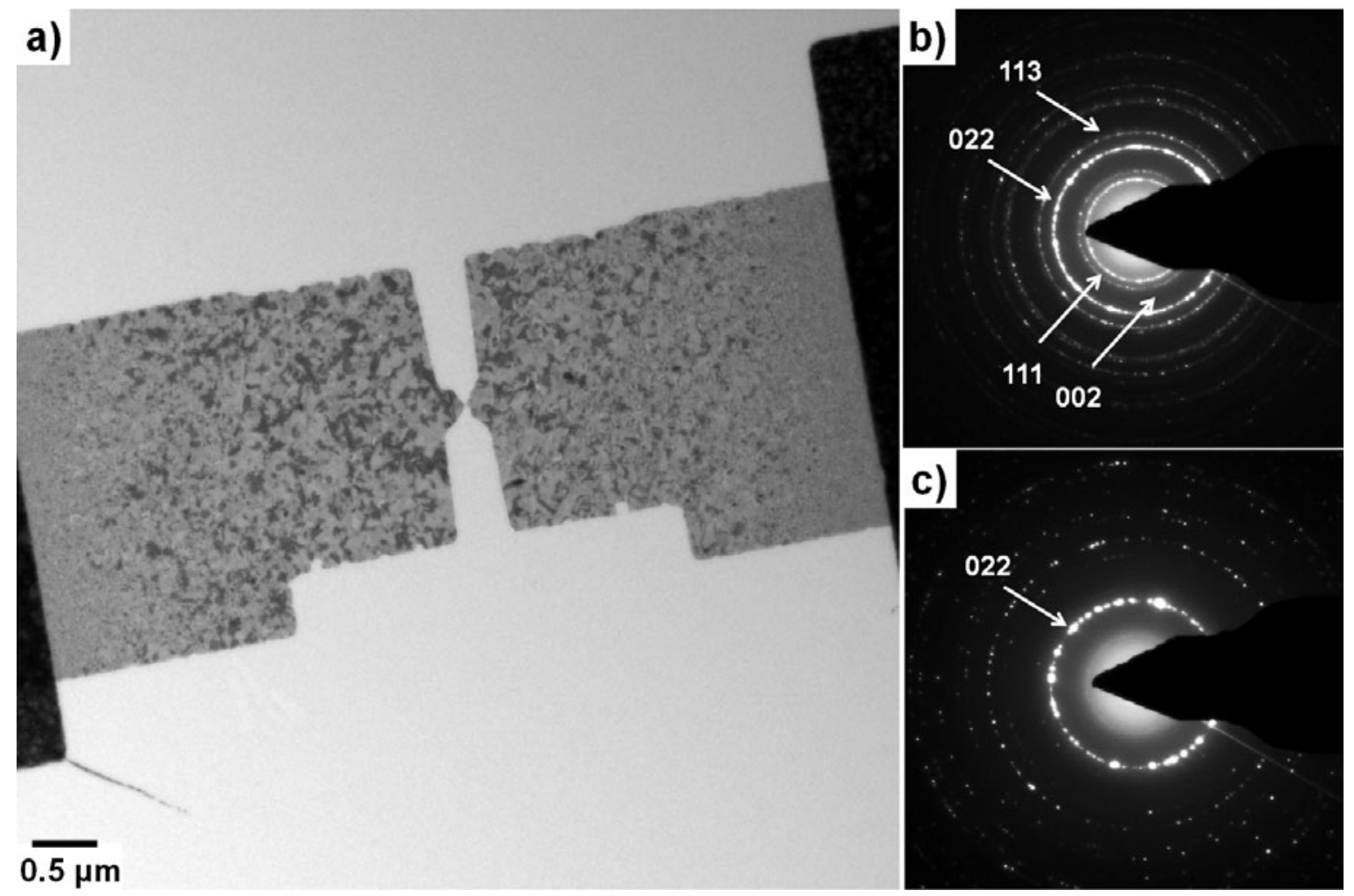

Figure 3. Distribution of recrystallization in a platinum $(\mathrm{Pt})$ nanowire. a: Broken nanowire in the center and leads to this bridge. Both were $14 \mathrm{~nm}$ thick. The black areas on both sides are Au contact lines. The dark contrast seen is due to recrystallized Pt. b,c: Diffraction patterns taken at A and B, respectively. Area A has the same diffraction pattern as the original sample. Area B shows the presence of the only diffraction rings that occur for [111]-oriented grains.

before changes that can be related to electromigration. Obviously, the grain growth is due to a temperature rise in the Pt wire and its neighborhood as previously described (Gao et al., 2011). After this initially similar behavior, the changes related to the electromigration process occur differently for UVI and FBC.

The effect of electromigration for the UVI mode can be seen in Figures $1 \mathrm{~d}-1 \mathrm{~h}$. At first a bottleneck is formed in the anode side of the wire. In Figures $1 \mathrm{e}-1 \mathrm{~g}$, the bottleneck shifts along the wire from the anode to the cathode side. Finally, a gap is formed near the cathode side of the wire. The electrodes on both sides of the gap are asymmetric. In most cases, the anode side has a point-like shape and the cathode side is flat, but other shapes (e.g., two flat electrodes) were also observed, thus indicating that the shape also depends on the local grains and their orientation. Whereas the bottleneck begins roughly in the middle of the nanowire, the location of the final gap was found to be random along the wire and was sometimes even observed in the wider region that connects the nanowire with the lead. In all cases, the bottleneck moved toward the cathode side. A similar gap formation was observed for UVI electromigration of gold nanowires (Heersche et al., 2007). However, there the anode side was flat and the cathode side pointed. The difference can be related to the wind force direction. In gold, the charge carriers are electrons and therefore the electron wind force is dominant (Ho \& Kwok, 1989).
The effect of electromigration for the FBC mode can be seen in Figures $2 \mathrm{c}-2 \mathrm{f}$. The narrowing of the nanowire started almost in the middle of the nanowire and stayed there until the wire was broken. The resulting gap is symmetric. Note that it is possible to correlate changes in the shape of the wire with applied current (see Fig. 2a). As can be seen in Figure 2, each loop on the I-V characteristic corresponds to the particular shape on the bridge.

The crystal orientations in the Pt nanowires before and after electromigration were studied. The wire initially has small grains with random orientation. After the recrystallization and electromigration processes, the nanowires contain larger grains. Diffraction patterns taken from the two different areas labeled A and B in Figure 3 show that the $002,022,111$, and 113 reflections are present in the nonrecrystallized area (Fig. 3b). During recrystallization, all reflections except those corresponding to the [111] orientation disappear. (Fig. 3c).

The self-breaking technique is widely used to prevent the formation of the metal particles in a gap during electromigration. It has been demonstrated that when using the conventional electromigration technique, regardless of FBC or UVI, there is always a chance that metal particles will be left in the gap. These particles can emit spurious signals. The self-breaking technique, which uses the spontaneous breaking of a metal junction $(<10 \mathrm{~nm})$ owing to the stress created in the junction during electromigration, can largely 


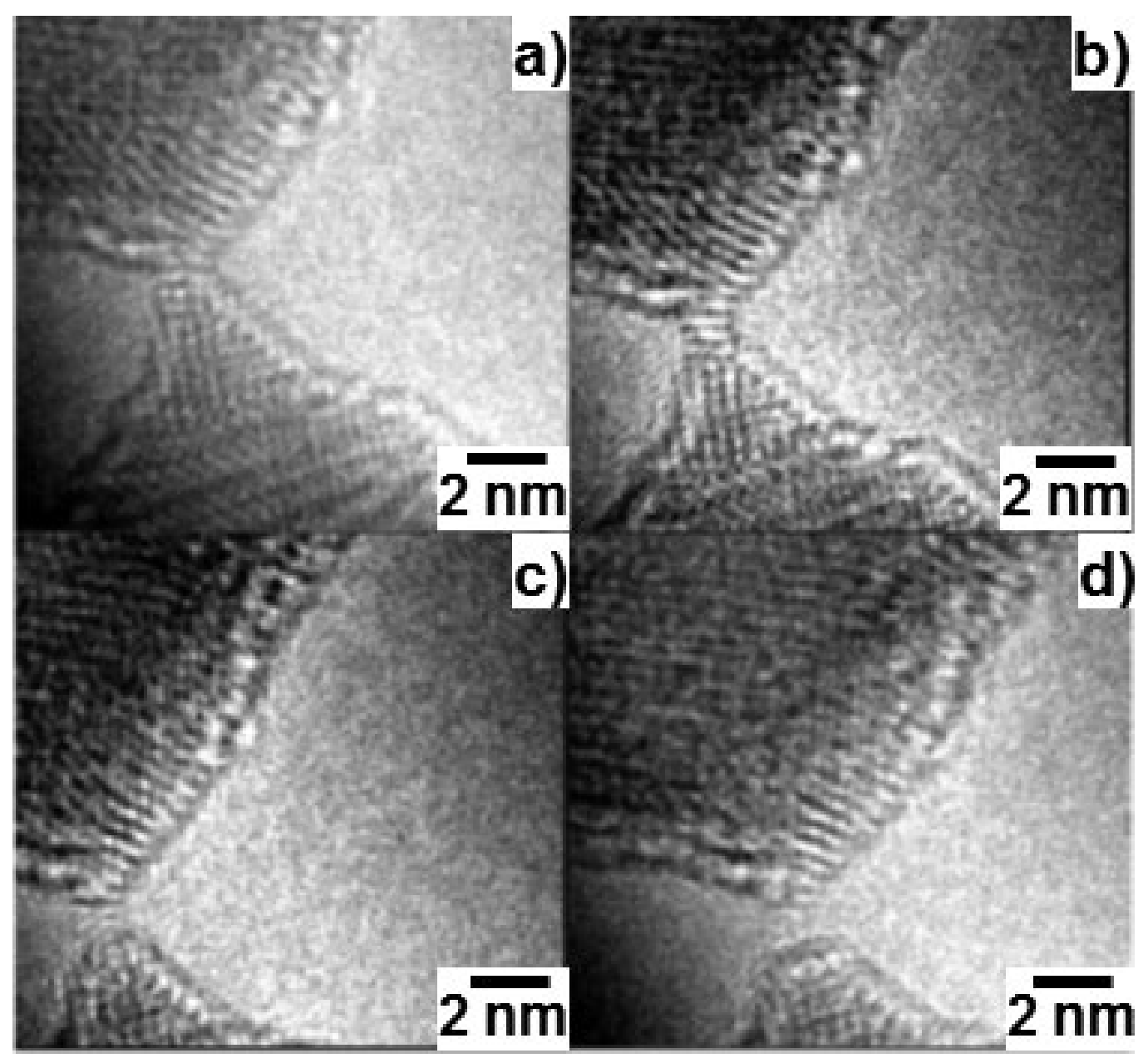

Figure 4. a-d: Self-breaking of a platinum junction. Images (b), (c), and (d) were taken 150, 200, and $750 \mathrm{~ms}$ after image (a).

solve the problem. In the case of gold nanowires, it is hard to image the self-breaking process because gold atoms are too mobile under the e-beam illumination. Owing to the higher stability of the Pt junction compared with that of gold, it is possible to image the self-breaking of the $\mathrm{Pt}$ junction with TEM. Figure 4 reveals the evolution of a $\mathrm{Pt}$ nanojunction from a few atoms of contact (Figs. 4a, $4 \mathrm{~b}$ ) to a single-atom contact (Fig. 4c), and finally to an open gap (Fig. 4d). The image rate is $50 \mathrm{~ms} /$ frame, from which we can estimate that the self-breaking takes place on a time scale of hundreds of milliseconds.

Previous papers on Au have provided a description of the $2 \mathrm{D}$ geometry of the gap, but information on the third dimension is still missing. High-angle annular darkfield (HAADF) imaging can provide such information. The HAADF signal is proportional to the mass thickness of the sample. In our case, the silicon nitride membrane is so thin that it emits only a weak background signal. After subtracting this background signal, the HAADF signal is then proportional to the thickness of the Pt electrodes. Several dark-field STEM images of nanogaps with FBC mode were recorded. Figure 5 shows a typical example of this and the corresponding height profile across the 4-nm-wide gap. The height profile was acquired by taking a 10-pixel-wide line profile across the nanogap at the position of the white line. Considering that the film thickness is $14 \mathrm{~nm}$, the thickness of the Pt electrode normally drops to 14 to $0 \mathrm{~nm}$ over a length of 5-10 nm. Figures $5 \mathrm{~b}$ and $5 \mathrm{c}$ show a $3 \mathrm{D}$ representation of this nanogap, which suggests that the narrowest part of the gap is on the silicon nitride membrane. Figure $5 \mathrm{c}$ is an enlargement of the gap part of Figure 5b. It shows the steep edges of the electrodes near the gap. The electrodes that are very close to the nanogap are only a few atoms thick. The other nanogaps imaged by dark-field STEM showed very similar profiles.

\section{Discussion}

The in situ TEM electromigration experiments show clear differences between the two current control procedures. In particular, the final results are very different. The gap is asymmetrical and symmetrical for UVI and FBC, respectively, and the location of the gap is reliably in the middle of the Pt nanowire for FBC, whereas it is at a random position for UVI. 


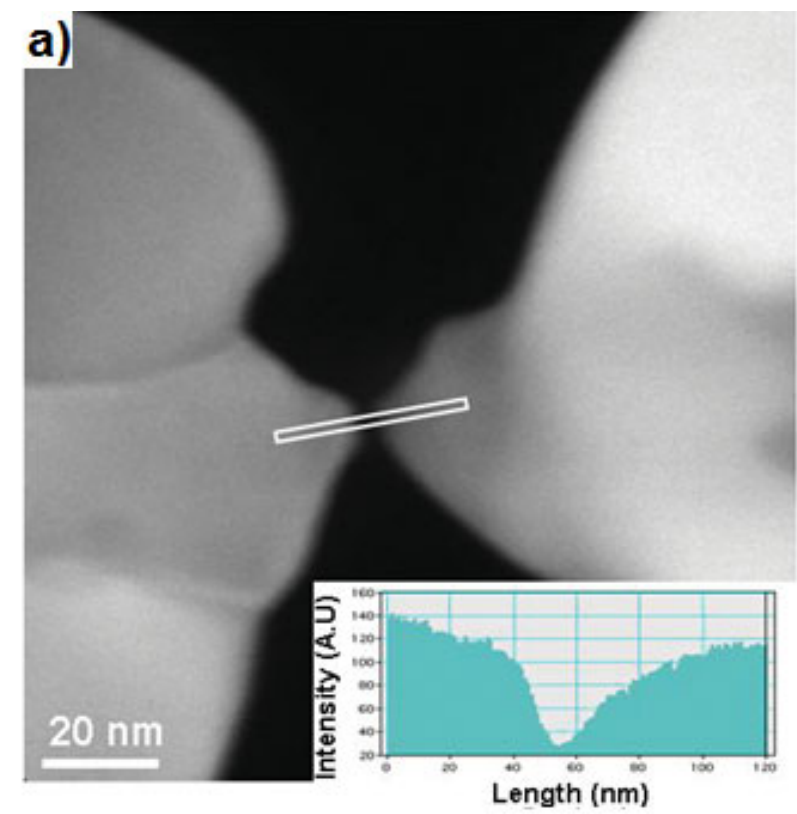

b)

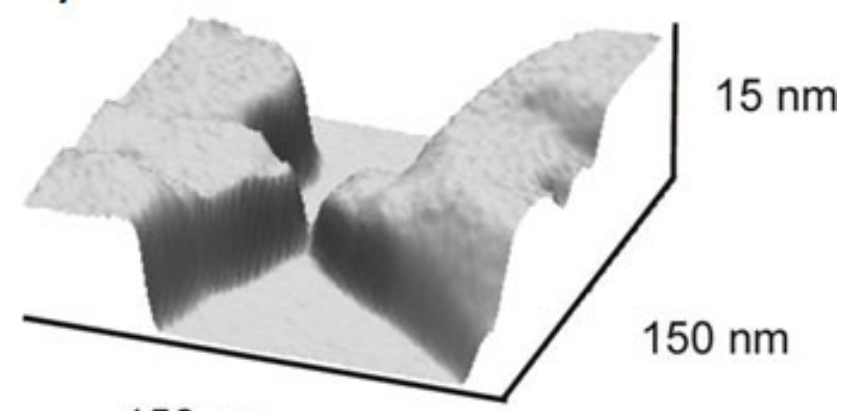

$150 \mathrm{~nm}$

Figure 5. a: Scanning transmission electron microscopy (STEM) image of a nanogap formed using feedback control electromigration. The white rectangle defines the area in which the intensity has been integrated. The inset shows the height profile of the broken nanowire across the gap. The thickness of the electrode evolves from nearly 0 to $14 \mathrm{~nm}$, which is the original thickness of the platinum nanowire. $\mathbf{b}$ : Three-dimensional nanogap profile reconstructed from the STEM image in (a).

The position of the gap in the middle of the nanowire for the FBC is quite understandable. The narrowing of the nanowire by electromigration will start close to the place where it is the hottest, which will be the middle of the nanowire. Once a constriction has formed, the resistance will increase and the current is dropped to zero in the FBC procedure. Although the current is swept up again, it is considerably smaller (see Fig. 2a) than $I_{\max }$ for the previous loops, because the new maximum current (set by feedback control) is tuned to the new size of the constriction. In the low-current part of the cycle, the local temperature at the bridge decreases. Tuning up the current will lead again to Joule heating, which will have the greatest influence at the constriction. Thus, the further narrowing is very likely to occur at the constriction. This cycle of the voltage increasing and dropping down to zero is repeated until a gap is formed.
The UVI procedure is identical to the FBC procedure up to the formation of the constriction. Thus, it is logical and indeed observed that the location of the constriction is the same as for FBC: it is approximately in the middle of the nanowire. When this constriction is formed, the current is further increased in the UVI procedure and thus the local temperature will increase. We observed that, once the constriction has formed, it moves in most cases toward the cathode side, but the speeds and paths vary greatly. This indicates that the local temperatures, as well as the grain sizes, orientations, and boundaries, all play an important role, making the further evolution of the electromigration process specimen dependent. It is known that grain boundary diffusion depends very much on the type of grain boundary (Smoluchowski, 1952). The higher temperatures of UVI (compared with FBC) during the electromigration process result in a different evolution of the gap, because the ratio between diffusion along the surfaces and the grain boundaries is different. It has been reported that at high temperatures $(1,600 \mathrm{~K})$ the Pt grain boundary diffusivity coefficient surpasses the surface diffusivity coefficient (Tu, 2003). Thus, in the case of UVI, the gap formation can vary significantly from wire to wire owing to the differences in grain boundaries and the difference in temperature gradient. Obviously, further research is needed to enable us to produce a detailed model of the constriction displacement and the gap formation in relation to the grains and grain boundaries in the nanowires.

Analysis of several HAADF STEM images of samples electromigrated with the FBC mode show roughly the same height profile as that indicated in Figure 5a. We used FBC only because it provides reproducibly two symmetrical pointy contacts instead of asymmetrical ones for the UVI. The advantage of having two pointy contacts is that it yields less shielding of the back gate and can thus be used for electrical measurements on single molecules. The knowledge of the 3D shape of the electrodes allows a detailed description of the electron tunneling processes across the gap. In this respect, it would be useful to perform in situ TEM imaging while simultaneously measuring the tunnel conductance. These measurements could then be used to test the validity of the different tunnel models and to obtain an accurate value of the barrier height in these junctions.

\section{SUMMARY}

In conclusion, we have performed an in situ TEM inspection of electromigration in Pt wires. Our study reveals the different gap formations for feedback-controlled versus noncontrolled electromigration. We also recorded for the first time a video showing the self-breaking process in Pt nanowires and demonstrated that HAADF STEM allows the determination of the 3D profile made by the electrodes via electromigration. Exact knowledge of the geometry of a tunneling junction makes it possible to validate various tunneling models. 


\section{ACKNOWLEDGMENTS}

This research was supported by the "NIMIC" project and the EU FP7 programme under the grant agreement "SINGLE."

\section{References}

Esen, G. \& Fuhrer, M.S. (2005). Temperature control of electromigration to form gold nanogap junctions. Appl Phys Lett 87, 263101-1-3.

Gao, B., Osorio, E.A., Babaei Gaven, K. \& van der Zant, H.S.J. (2009). Three-terminal electric transport measurements on gold nano-particles combined with ex situ TEM inspection. Nanotechnology 20, 415207.

Gao, B., Rudneva, M., McGarrity, K.S., Xu, Q., Prins, F., Thijssen, J.M., Zandbergen, H. \& van Der Zant, H.S. (2011). In situ transmission electron microscopy imaging of grain growth in a platinum nanobridge induced by electric current annealing. Nanotechnology 22(20), 205705.

Grose, J.E., Tam, E.S., Timm, C., Scheloske, M., Ulgut, B., Parks, J.J., Abrua, H.D., Harneit, W. \& Ralph, D.C. (2008). Tunnelling spectra of individual magnetic endofullerene molecules. Nat Mater 7, 884-889.

Heersche, H.B., Lientschnig, G., O’Neill, K., van der Zant, H.S.J. \& ZandBergen, H.W. (2007). In situ imaging of electromigration-induced nanogap formation by transmission electron microscopy. Appl Phys Lett 91, 072107-1-3.

Ho, P.S. \& Кшок, Т. (1989). Electromigration in metals. Rep Prog Phys 52, 301-348.

Houck, A.A., Labaziewicz, J., Chan, E.K., Folk, J.A. \& Chuang, I.L. (2005). Kondo effect in electromigrated gold break junctions. Nano Lett 5(9), 1686-1688.
O’Neill, K., Osorio, E.A. \& van der Zant, H.S.J. (2007). Selfbreaking in planar few-atom Au constrictions for nanometerspaced electrodes. Appl Phys Lett 90, 133109-1-3.

Park, H., Lim, A.K.L., Alivisatos, A.P., Park, J. \& McEuen, P.L. (1999). Fabrication of metallic electrodes with nanometer separation by electromigration. Appl Phys Lett 75, 301-303.

Prins, F., Hayashi, T., de Vos van Steenwijk, B.J.A., Gao, B., Osorio, E.A., Muraki, K. \& van der Zant, H.S.J. (2009). Room-temperature stability of Pt nanogaps formed by selfbreaking. Appl Phys Lett 94, 123108-1-3.

Smoluchowski, R. (1952). Theory of grain boundary diffusion. Phys Rev 87(3), 482-487.

Strachan, D.R., Johnston, D.E., Guiton, B.S., Datta, S.S., Davies, P.K., Bonnell, D.A. \& Charlie Johnson, A.T. (2008). Real-time TEM imaging of the formation of crystalline nanoscale gaps. Phys Rev Lett 100, 056805-1-4.

Strachan, D.R., Smith, D.E., Johnston, D.E., Park, T.-H., TheRIEN, M.J., BonnelL, D.A. \& Johnson, A.T. (2005). Controlled fabrication of nanogaps in ambient environment for molecular electronics. Appl Phys Lett 86, 043109-1-3.

Taychatanapat, T., Bolotin, K.I., Kuemmeth, F. \& Ralph, D.C. (2007). Imaging electromigration during the formation of break junctions. Nano Lett 7 (3), 652-656.

Tu, K.N. (2003). Recent advances on electromigration in very-largescale-integration of interconnects. J Appl Phys 94, 5451-5473.

van der Zant, H.S.J., Kervennic, Y., Рoot, M., O’Neill, K., De Groot, Z., Thijssen, J.M., Heersche, H.B., Stuhr-Hansen, N., BjDørnholm, T., Vanmaekelbergh, D., van Walree, C.A. \& JeNNESKens, L.W. (2006). Molecular three-terminal devices: Fabrication and measurements. Faraday Discuss 131, 347-356. 